\title{
Analysis and Modeling of an Electrostatic Induction Micromotor
}

\author{
*F. J. Santana Martín, **S. García-Alonso Montoya, *J. M. Monzón Verona, **J. A. Montiel-Nelson. \\ *Electrical Eng. Dept., University of Las Palmas de Gran Canaria. \\ **Electronic and Automation Eng. Dept., University of Las Palmas de Gran Canaria. \\ E-mails: fsantana@die.ulpgc.es, sgarcia@diea.ulpgc.es,jmonzon@die.ulpgc.es,montiel@iuma.ulpgc.es
}

\begin{abstract}
An electrostatic induction micromotor has been simulated and analyzed using Maxwell equations defining the mathematical model that governs it. We have introduced the analytical solution of the mathematical model for a simple geometry of the micromotor. The obtained results have been generalized for a model that presents cylindrical symmetry. An approximate solution has been evaluated through the realization of the analysis and numeric calculus (FEM) of the model. The force density has been estimated using the tensorial calculus. The FEM solution has been compared with the analytical solution for verification purpose.
\end{abstract}

\section{INTRODUCTION}

The present paper deals with the design, simulation and analysis of an electrostatic induction micromotor. The laws that govern the operation of electromagnetic induction motors used in the industry are well known. However, micro scale effects should be considered for micromotor design and implementation. These effects are discussed in this paper.

Currently, the design and implementation of a micromotor using MEMS technology is a great challenge. For this purpose, we have developed some tools based on FEM to simulate the electromagnetic fields and force density of an electrostatic micromotor. The proposed analytical equations are compared with the obtained solutions provided by FEM tools. To our knowledge, only a few publications deal with this topic [1]-[3].

The study has been carried out in a simple linear electrical induction micromachine constituted by two parallel plates rotor and stator- isolated by a dielectric [4]. The distance between plates is $6 \mu \mathrm{m}$. Fig. 1 illustrates the geometry and electromechanical coupling effects of a linear electrical induction micromachine.

This work is focused in the linear micromachine due to the greater simplicity of its analytical equations. The linear micromachine is the unfolding of a rotating electric micromachine, and this is the reason why the conclusions obtained for the linear micromachine are easily generalized to the rotating one.

\section{PHYSICAL PRINCIPLES}

The main principles that explain micromachines in the microscale are different from those that are present in the macroscale. The Paschen's [5] law applied in the microscale establishes that the electric field intensity $E$ roles the behaviour of the electrostatic induction micromachines, and not the magnetic field intensity, as in the macroscale. Paschen's law can be expressed as

$$
E_{B}=100 \frac{365 p}{1.18+\ln (p d)} \quad(\mathrm{V} / \mathrm{cm} \text { Torr })
$$

where, parameters $p$ and $d$ are introduced in Table I. Fig. 2 illustrates the electric field vs. distance for Paschen's law.

TABLE I

SYMBOLS

\begin{tabular}{|c|c|c|}
\hline Symbol & Name & Units \\
\hline$a$ & Height of dielectric 2 & $\mathrm{~m}$ \\
\hline$d$ & Distance & $\mathrm{cm}$ \\
\hline$E_{B}$ & Break electric field & $\mathrm{V} / \mathrm{m}$ \\
\hline$k$ & Number of waves per metre & - \\
\hline$j$ & Imaginary unity & - \\
\hline$J_{f}$ & Volumetric current density & $\mathrm{A} / \mathrm{m}^{2}$ \\
\hline$p$ & Pressure & Torr \\
\hline$S$ & Slip & - \\
\hline$v$ & Linear speed of mobile part & $\mathrm{m} / \mathrm{s}$ \\
\hline$V$ & Interelectrodic voltage & $\mathrm{V}$ \\
\hline$V_{0}$ & Supply voltage & $\mathrm{V}$ \\
\hline$E_{0}$ & Permittivity vacuum & $\mathrm{F} / \mathrm{m}$ \\
\hline$\varepsilon_{a}$ & Electric permittivity of the dielectric & $\mathrm{F} / \mathrm{m}$ \\
\hline$\varepsilon_{e f f}$ & Effective permittivity & $\mathrm{F} / \mathrm{m}$ \\
\hline$\varphi$ & Electric scalar potential & $\mathrm{V}$ \\
\hline$\omega$ & Signal frequency & $\mathrm{Hz}$ \\
\hline$\sigma_{a}$ & Electric conductivity of the dielectric & $\mathrm{S} / \mathrm{m}$ \\
\hline$\sigma_{e f f}$ & Effective Conductivity & $\mathrm{S} / \mathrm{m}$ \\
\hline$\sigma_{f}$ & Surface current density & $\mathrm{A} / \mathrm{m}$ \\
\hline$\sigma_{S}$ & Resistivity & $1 / \Omega$ \\
\hline$\Phi^{b}$ & Voltage in the interface & $\mathrm{V}$ \\
\hline$\rho_{f}$ & Volumetric charge density & $\mathrm{C} / \mathrm{m}^{3}$ \\
\hline & & \\
\hline
\end{tabular}

We have studied the energy density in both macroscale and microscale level. The electric energy density in the microscale is very high compared with the electromagnetic energy density in the same scale. This is the opposite that in the macroscale. Besides, we have studied the electric force per unit of volume (electrostatic force density) in the microscale for our micromachine and its expression is as follows:

$$
F_{v}^{m}=-\frac{\varepsilon_{0} V^{2}}{2 \mathrm{~d}^{3}} \quad\left(\mathrm{~N} / \mathrm{m}^{3}\right)
$$




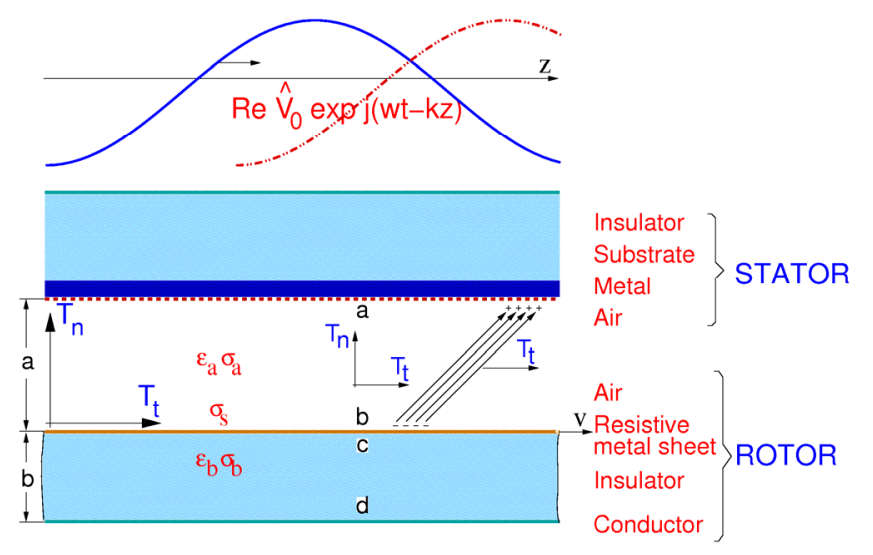

Fig. 1. Linear electrical induction micromachine.

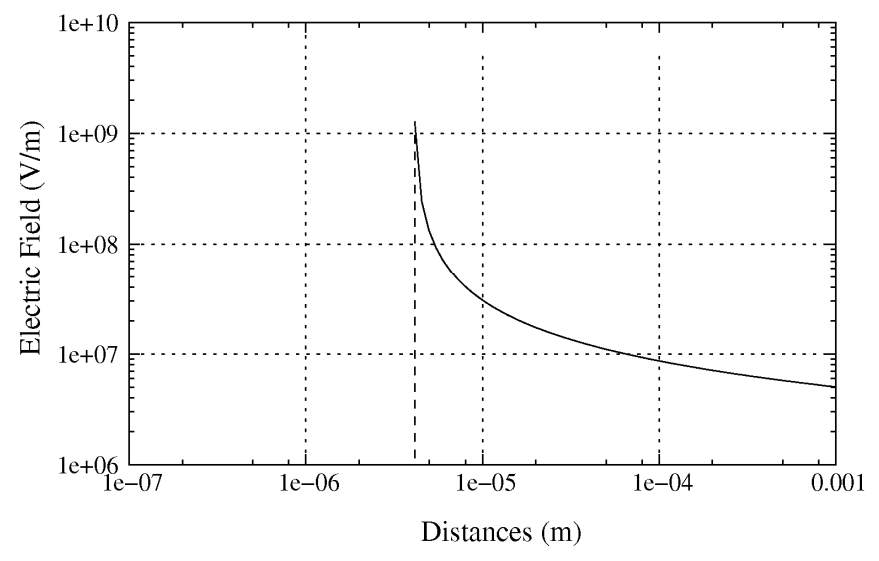

Fig. 2. Graphical representation of Paschen's law.

It can be seen that for a particular voltage $V$, the lower the distance, the higher the electric density force. This expression has been deduced based on the energy stored in a planar capacitor.

On the other hand, we have also calculated the electromagnetic energy density in the macroscale which is expressed as

$$
F_{v}^{M}=J B \quad\left(\mathrm{~N} / \mathrm{m}^{3}\right)
$$

Where, $J$ is the current density and $\mathrm{B}$ is the magnetic flux density.

Equations (2) and (3), electrostatic force density in the microscale and electromagnetic in the macroscale, respectively, indicates that the energy density is greater in the microscale.

One important advantage of induction electrostatic micromachines is they needn't coils for their operation. Besides, the traditional techniques of fabrication of integrated circuits can be implemented for its manufacturing.

\section{ANALYTICAL EQUATIONS}

From the basic physical principles that govern the micromotor behaviour, we determined an analytical equation for a planar elemental model, as is expressed in (4).

$\left\langle T_{z}\right\rangle_{z}$ represents the mean force density of the micromachine.

$\left\langle T_{z}\right\rangle_{z}=\frac{1}{2}\left(k V_{0}\right)\left(k V_{0}\right) \varepsilon_{a}\left(\varepsilon_{a} \sigma_{e f f-} \varepsilon_{e f f} \sigma_{a}\right) \frac{S_{E}}{1+S_{E}^{2}} K\left(\mathrm{~N} / \mathrm{m}^{2}\right)$

where, $K$ stands for

$$
K=\frac{1}{\varepsilon_{e f f} \sigma_{e f f} \sinh ^{2}(k a)}
$$

and, $S_{\mathrm{E}}$ stands for

$$
S_{E}=\tau_{E} \omega S
$$

$S$ is the slip and $\tau_{E}$ stands for

$$
\tau_{E}=\frac{\varepsilon_{e f f}}{\sigma_{e f f}}
$$

All the parameters and variables were introduced in Table I.

Fig. 3 shows the graphic representation of the analytic expression for the force density presented in (4). Slip $S$ is the independent variable. The force density has been represented in this case for a value of the resistivity $\sigma_{S}=1 /\left(2 \times 10^{8}\right)(1 / \Omega)$.

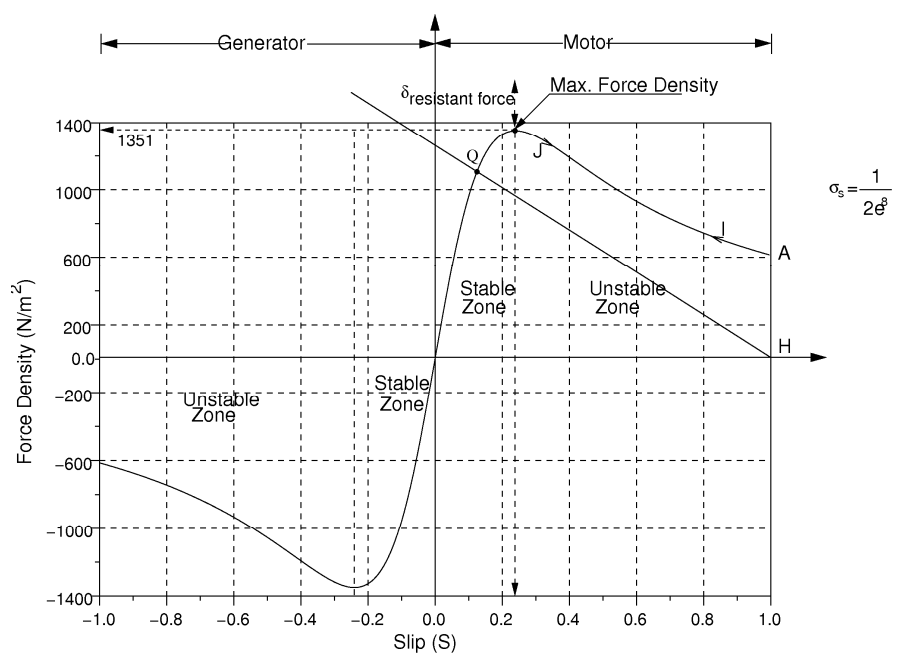

Fig. 3 Static working point.

At the same figure, we have also represented the resistant force density which is applied to the micromotor in a particular moment. This force resistant has been assumed linear and, therefore, its representation is a straight line.

In order to calculate the force density through the electrostatic field and the induced charge in the mobile part of the micromachine, first, the potential in the interface must be evaluated. To accomplish this task we apply the charge 
Proceedings of the 2008 International Conference on Electrical Machines

conservation law in the interface. We start from Laplace equation

$$
\nabla^{2} \phi=0
$$

This equation has been particularized for the following boundary conditions: zero volts for the inferior plate of the mobile part and $\mathrm{V}$ volts for the fixed part.

By this way, we obtain next equation

$$
\frac{\partial \sigma_{f}}{\partial t}+\nabla_{S} \cdot\left(\sigma_{S} \cdot \vec{E}_{Z}+\vec{v}_{Z} \cdot \sigma_{f}\right)+\vec{n} \cdot\|\sigma \vec{E}\|=0
$$

Once we have developed the terms of this equation, we obtain the voltage in the interface of the micromotor

$$
\widehat{\Phi}^{b}=\frac{V_{0}}{\sinh (k a)} \frac{\frac{\sigma_{a}}{\sigma_{e f f}}+\frac{\varepsilon_{a}}{\varepsilon_{e f f}} \omega S j}{\left(1+\frac{\varepsilon_{e f f}}{\sigma_{e f f}} \omega S j\right)}
$$

where,

$$
\begin{gathered}
\sigma_{e f f}=\sigma_{a} \operatorname{coth}(k a)+\operatorname{coth}(k b) \sigma_{b}+\sigma_{S} k \\
\varepsilon_{e f f}=\varepsilon_{a} k \operatorname{coth}(k a)+\varepsilon_{b} k \operatorname{coth}(k b)
\end{gathered}
$$

Please, note that parameters and variables were introduced in Table I.

\section{FEM ANALYSIS}

To our knowledge, no FEM analysis has been found in the literature for the induction micromotor. Finite element methods are used when the model introduces an irregular geometry and the analytical solution becomes nearly impossible to be obtained or, when the material properties are changed due to the anisotropy of the medium.

The first step in a FEM analysis is to determine the field equation that defines the problem. The following equations are referenced in [4] and [7]-[9], and they have been taken as the base for this work.

As initial assumption we use Gauss's law:

$$
\nabla \cdot \varepsilon \vec{E}=\rho_{f}
$$

The charge conservation law says:

$$
\nabla \cdot \vec{J}_{f}+\frac{\partial \rho_{f}}{\partial t}=0
$$

And the constitutive law:

$$
\vec{J}_{f}=\sigma \vec{E}+\vec{v} \rho_{f}
$$

As initial hypothesis we assume a quasi conservative electric field, therefore

$$
\begin{gathered}
\nabla \times \vec{E} \approx 0 \\
\nabla \cdot \sigma \vec{E}+\nabla \cdot \rho_{f} \vec{v}+\frac{\partial \rho_{f}}{\partial t}=0
\end{gathered}
$$

The free charge volumetric density is eliminated taking into account (13), so

$$
\nabla \cdot \sigma \vec{E}+\nabla \cdot(\vec{v} \nabla \cdot \varepsilon \vec{E})+\frac{\partial}{\partial t} \nabla \cdot \varepsilon \vec{E}=0
$$

In the same way, taking into account that

$$
\vec{E}=-\nabla \varphi
$$

we eliminate $E$ from (18) and we obtain next equation

$$
\nabla \cdot \sigma \nabla \phi+\nabla \cdot(\vec{v} \nabla \cdot \varepsilon \nabla \phi)=-\frac{\partial}{\partial t} \nabla \cdot \varepsilon \nabla \phi
$$

At the start, $v=0$, then

$$
\nabla \cdot \sigma \nabla \phi+\frac{\partial}{\partial t} \nabla \cdot \varepsilon \nabla \phi=0
$$

The previous equation is expressed in the time domain. In sinusoidal stationary regimen, the operator $\partial / \partial t$ is equal to $j \omega$ and it is expressed in the following form

$$
\nabla \cdot \sigma \nabla \bar{\phi} e^{j \omega t}+j \omega \nabla \cdot \varepsilon \nabla \bar{\phi} e^{j \omega t}=0
$$

where $\bar{\phi}$ is a special complex distribution. By simplifying the time dependent term $e^{j \omega t}$, we obtain (23). This field equation represents the behavior of the structure in which we are going to apply the FEM analysis.

$$
\nabla \cdot \sigma \nabla \bar{\phi}+j \omega \nabla \cdot \varepsilon \nabla \bar{\phi}=0
$$

In this equation the scalar potential $\bar{\phi}$ is the unknown variable.

The two main methods derived from the FEM equations are the variational approximation and the Galerkin approximation that is a special case of the weighted residue method (WRM), [11]-[16].

The variational method was first applied to magnetic problems and embraced the major part of the scientific literature. But nowadays, due to its generality, Galerkin's method is increasing its popularity and it is the method we have used in this work.

The WRM requires that the integral of the projection of the residue on a specific weight function is cero in the domain of interest. The selected weight function determines the type of WRM.

In this work, we have chosen a weight function that has the same form of the shape functions of finite elements. This is the method known as Galerkin's method. 
Equation (23) has been discretized and Galerkin [10] method has been applied on the plane domain of the discretized micromotor.

Five different regions have been considered for discretization: the metal plate of the fixed element, the dielectric (air), and the resistive metal sheet, the dielectric (insulator) and the metal plate (conductor) of the mobile part, as can be seen in Fig. 1. For this discretization triangular first order isoparametric elements have been used.

A classical problem in rotating and linear machines is that the meshing of the regions is different in each step of the calculus. To avoid this problem, we work with only one invariable mesh and we introduce the speed factor in the formulation of the potential in each node, as can be seen in (24). In addition, this saves a great amount of time.

$$
\Phi^{b}=\frac{V_{0}}{\sinh (k a)} \frac{\frac{\sigma_{a}}{S}+\varepsilon_{a} \omega j}{\frac{\sigma_{e f f}}{S}+\varepsilon_{e f f} \omega j}
$$

Equation (24) represents the value of the electric potential as a function of the material conductivity that is modified by the speed factor - it is contained in the slip term $S$.

The structure that we analyze has an elemental cell that is repeated in the $\mathrm{Z}$ direction as can be seen in Fig. (4). This simplifies noticeable the numerical calculus.

Fig. 5 shows the non-null elements in the stiffness matrix obtained when solving a finite element problem. The matrix has 2407 rows and 2407 columns, and there are 15586 non-null elements, with a non-null element density of $0.269 \%$, which is very low. The stiffness matrices we are working with are clearly sparse matrices.

\section{RESULTS}

We have calculated the potential in the interface applying FEM and the obtained analytical equations (see (23)) for five different values of the conductivity. The error between the results obtained using analytical equations and the FEM are neglected, as is shown in Table II. Fig. 6 shows the FEM results for a conductivity of $1 /\left(600 \cdot 10^{6}\right)(\mathrm{S} / \mathrm{m})$.

Typical maximum discrepancies are lower than $0.1 \%$ as is shown in Table II. This table represents the electric voltage in permanent regimen in the interface.

We have also calculated the electric field in the interface. FEM results and analytical solution results are presented in Table III. Fig. 7 shows FEM results for a conductivity of $1 /\left(600 \cdot 10^{6}\right)(\mathrm{S} / \mathrm{m})$.

The error between the results obtained using analytical equations and the finite element method are also neglected.

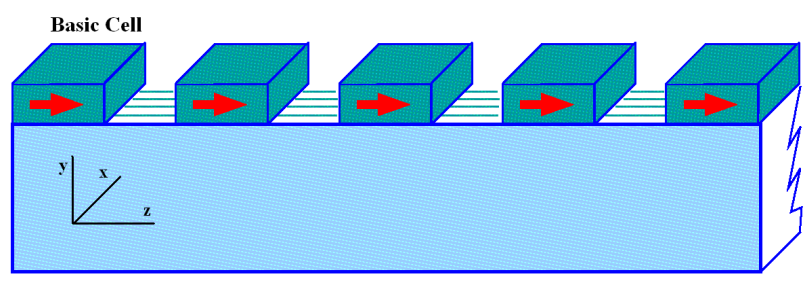

Fig. 4. Periodic boundary conditions

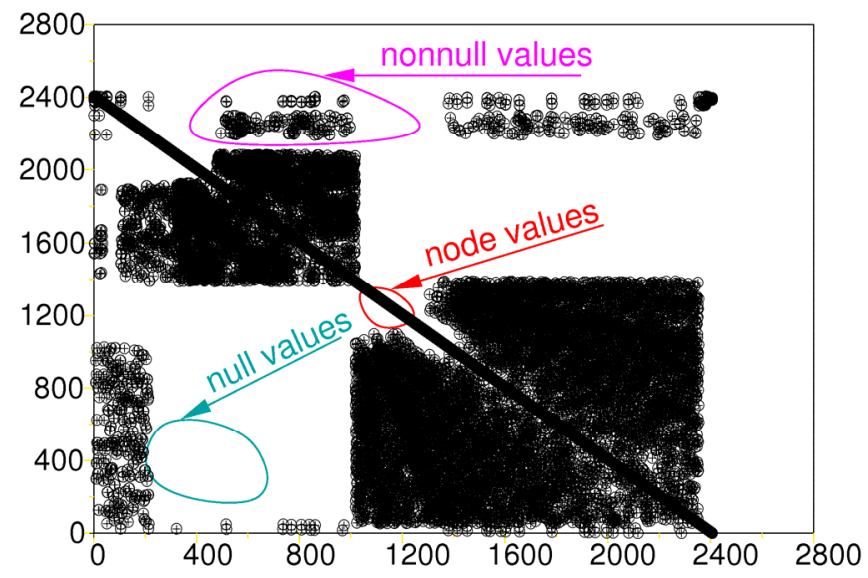

Fig. 5. Representation of the stiffness matrix for a FEM solution.

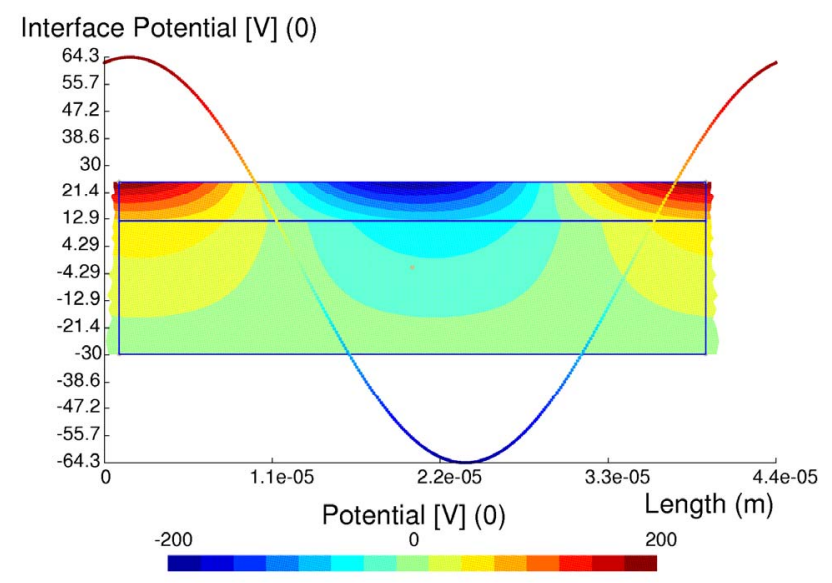

Fig. 6. Graphical representation of FEM solution in all the domain and potential in the interface mobile part-air.

TABLE II

INTERFACE ELECTRICAL VOLTAGE

\begin{tabular}{|c|c|c|c|}
\hline $\begin{array}{c}\text { Conductivity } \\
(\mathbf{1} / \mathbf{\Omega})\end{array}$ & Analytical & FEM & $\begin{array}{c}\text { Error } \\
\mathbf{( \% )}\end{array}$ \\
\hline $1 /\left(50 \cdot 10^{6}\right)$ & 21.6688 & 21.6947 & -0.119 \\
\hline $1 /\left(100 \cdot 10^{6}\right)$ & 37.7909 & 37.7259 & 0.172 \\
\hline $1 /\left(200 \cdot 10^{6}\right)$ & 53.6311 & 53.5904 & 0.075 \\
\hline $1 /\left(600 \cdot 10^{6}\right)$ & 64.2738 & 64.2748 & -0.001 \\
\hline $1 /\left(1800 \cdot 10^{6}\right)$ & 65.8906 & 65.9102 & -0.029 \\
\hline
\end{tabular}




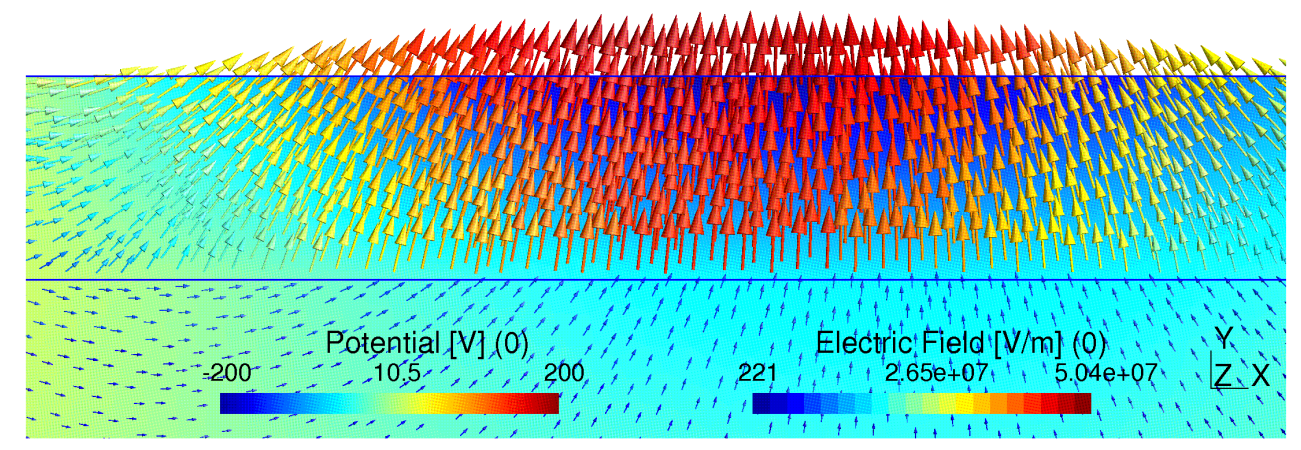

Fig. 7. Electric field in the interface for a conductivity of $1 /\left(600 \cdot 10^{6}\right)(\mathrm{S} / \mathrm{m})$.

TABLE III

ELECTRIC FIELD (V/m) IN THE STEADY STATE IN THE INTERFACE IN Z=0

\begin{tabular}{|c|c|c|c|}
\hline $\begin{array}{c}\text { Conductivity } \\
(\mathbf{1} / \mathbf{\Omega})\end{array}$ & $\begin{array}{c}\text { Analytical } \\
\text { solution } \\
(\mathbf{V} / \mathbf{m})\end{array}$ & $\begin{array}{c}\text { Numeric } \\
\text { solution } \\
(\mathbf{V} / \mathbf{m})\end{array}$ & $\begin{array}{c}\text { Error } \\
\mathbf{( \% )}\end{array}$ \\
\hline $1 /\left(50 \cdot 10^{6}\right)$ & 3094307 & 3102000 & -0.248 \\
\hline $1 /\left(100 \cdot 10^{6}\right)$ & 5381641 & 5389700 & -0.149 \\
\hline $1 /\left(200 \cdot 10^{6}\right)$ & 7658503 & 7665400 & -0.090 \\
\hline $1 /\left(600 \cdot 10^{6}\right)$ & 9178278 & 9182800 & -0.049 \\
\hline $1 /\left(1800 \cdot 10^{6}\right)$ & 9409100 & 9419900 & -0.114 \\
\hline
\end{tabular}

FEM convergence has been guaranteed with the refining of the meshes of the micromotor as is shown in Table IV. The electrical voltage in the interface has been obtained for a conductivity of $1 /\left(1800 \cdot 10^{6}\right)(\mathrm{S} / \mathrm{m})$.

TABLE IV

EFFECT OF THE MESH IN THE CONVERGENCE

\begin{tabular}{|c|c|c|c|c|}
\hline $\begin{array}{c}\text { Number of } \\
\text { nodes }\end{array}$ & $\begin{array}{c}\text { Number of } \\
\text { elements }\end{array}$ & $\begin{array}{c}\text { Analytical } \\
\text { solution } \\
(\mathbf{V})\end{array}$ & $\begin{array}{c}\text { Numeric } \\
\text { solution } \\
(\mathbf{V})\end{array}$ & $\begin{array}{c}\text { Error } \\
\mathbf{( \% )}\end{array}$ \\
\hline 2353 & 4704 & 65.89 & 65.91 & 0.030 \\
\hline 613 & 1224 & 65.89 & 66.02 & 0.197 \\
\hline 284 & 566 & 65.89 & 66.20 & 0.470 \\
\hline 170 & 338 & 65.89 & 66.40 & 0.774 \\
\hline
\end{tabular}

\section{CONCLUSION}

A mathematical model has been deduced for the induction electric lineal micromotor using the field equations. An exact analytical equation has been found. Using this equation the potential, the electrical field and the force density has been determined.

An analysis using FEM has been carried out and the same parameters (potential, electrical field and force density) have been calculated. Results have been compared and errors are neglected (lower than $0.1 \%$ ).

\section{ACKNOWLEDGMENT}

The authors gratefully acknowledge the collaboration of the IUMA (Institute for Applied Microelectronics of the University of Las Palmas de Gran Canaria, Spain).

\section{REFERENCES}

[1] C. Livermore et al.,"A High-Power MEMS Electric Induction Motor", Journal of Microelectromechanical Systems, vol. 13, n. 3, June 2004.

[2] N. Boukari, Y. Lefevre and P Spiteri, "Modeling the movement of electrostatic motors in 3D finite element code", IEEE Transactions on Magnetics., vol. 36, n. 4, July 2000.

[3] D. F. Busse, J. M. Erdman, R. J. Kerkman, D.W. Schlegel and G. L. Skibinski, "An evaluation of the electrostatic shielded induction motor", IEEE Trans. on Industry Applications, vol. 33, n. 6, NovemberDecember 1997.

[4] H. H. Woodson and J. R. Melcher, Electromechanical Dynamics Part III Elastic and Fluid Media, 1985.

[5] F. Paschen, "Ueber die zum funkenübergang in luft, wasserstof und kohlensäure bei verschiedenen drucken erforderliche potentialdifferenz," Annal der Physik, vol. 37, pp. 69-96, 1889.

[6] F.J Santana., J Montiel-Nelson., J.M. Monzón, “Análisis, Modelado y Optimización de un Micromotor de Inducción Electrostático", ETSIT, ULPGC, 2007.

[7] J. R. Melcher, Continuum Electromechanics, June 1981.

[8] H. H. Woodson and J. R. Melcher, Electromechanical Dynamics Part II Fields, Forces and Motion, 1985.

[9] H. H. Woodson and J. R. Melcher, Electromechanical Dynamics Part I Discrete Systems, 1985.

[10] O.C. Zienkiewicz and R.L. Taylor, El método de los elementos finitos, vol. 1, Sec. 9.4, p. 223.

[11] C.R.I. Emson, "The computation of electrostatic fields and space charge effects", IEE Colloquium on Computation in Electrostatics, Jan 1995.

[12] S. J. Salon, Finite element analysis of electrical machines.

[13] G. Li, and N. Aluru, "Efficient mixed domain analysis of electrostatic MEMS ", in Proc. Intl. Conf. on Computer Aided Design 2002, pp.474477, Nov. 2002.

[14] S. W. Chyuan, Y.-S. Liao and J.-T. Chen. "An efficient method for solving electrostatic problems" Computing in Science \& Engineering, vol.5, pp. 52-58, May-June 2003.

[15] D. Roger, H. C. Lai and P. Leonard, "Coupled elements for problems involving movement", IEEE Trans. on Magnetic, vol. 26, n. 2, March 1990.

[16] C.-Y. Hui, J.-L. Yeh and N. C. Tien, "Calculation of electrostatic forces and torques in MEMS using path-independent integrals", J. Micromech. Microeng, vol. 10, n. 3, pp. 477-482, 2000. 